\title{
Tilting Catalyst-Free InAs Nanowires by 3D-Twinning and Unusual Growth Directions
}

\author{
Heidi Potts, ${ }^{\dagger}$ Youri van Hees, ${ }^{\dagger, \dagger}$ Gözde Tütüncüoglu, ${ }^{\dagger}$ Martin Friedl, ${ }^{\dagger}$ Jean-Baptiste Leran, ${ }^{\dagger}$ \\ and Anna Fontcuberta i Morral* ${ }^{\dagger} \dagger$ (i) \\ ${ }^{\dagger}$ Laboratoire des Matériaux Semiconducteurs, École Polytechnique Fédérale de Lausanne, 1015 Lausanne, Switzerland \\ ${ }^{\ddagger}$ Institute of Applied Physics, Eindhoven University of Technology, 5600 MB Eindhoven, The Netherlands
}

Supporting Information

ABSTRACT: Controlling the growth direction of nanowires is of strategic importance both for applications where nanowire arrays are contacted in parallel and for the formation of more complex nanowire networks. We report on the existence of tilted InAs nanowires on (111)B GaAs. The tilted direction is predominantly the result of a three-dimensional twinning phenomenon at the initial stages of growth, so far only observed in VLS growth. We also find some nanowires

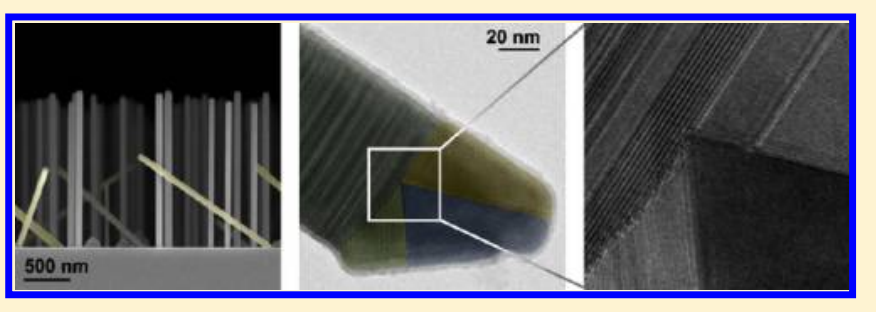
growing in $\langle 112\rangle$ and other directions. We further demonstrate how the tilting of nanowires can be engineered by modifying the growth conditions, and outline the procedures to achieve fully vertical or tilted nanowire ensembles. Conditions leading to a high density of tilted nanowires also provide a way to grow nanoscale crosses. This work opens the path toward achieving control over nanowire structures and related hierarchical structures.

\section{INTRODUCTION}

Semiconductor nanowires are promising candidates for future electronic, optoelectronic, and energy harvesting devices as well as a platform to investigate low-dimensional phenomena. ${ }^{1-8}$ In certain applications such as light emitting diodes or solar cells, it is highly desirable that nanowires are contacted in parallel on as-grown substrates. In this case, a complete control of the nanowire growth direction is essential in order to avoid leakage or open-circuit pathways. For other applications such as Majorana Fermion quantum computing, the growth direction determines the g-factor and spin-orbit interaction. In addition, any quantum logic involving Majorana Fermions requires more than one branch, e.g., nanowire crosses are desired. ${ }^{9-11}$

In general, III-V nanowires grow preferentially in the $\langle 111\rangle \mathrm{B}$ direction, resulting in mostly vertical nanowires when grown on (111)B substrates. Interest in nonconventional growth directions has increased significantly during the last years, and in the case of metal-catalyzed nanowires, a variety of different directions have been reported. ${ }^{12}$ Recent examples include $\langle 111\rangle \mathrm{A}$ oriented GaAs and $\mathrm{GaSb}$ nanowires, ${ }^{13,14}$ and InP nanowires for which the direction can be switched between $\langle 111\rangle \mathrm{B}$ and $\langle 100\rangle .{ }^{15}$ In addition, InAs nanowires in $\langle 001\rangle$ direction and $\langle 112\rangle$ direction have been demonstrated for goldcatalyzed MOVPE growth. ${ }^{16}$ The change in growth direction has been attributed to a change of contact angle of the liquid droplet in the vapor-liquid-solid (VLS) mechanism, or by dynamics at the growth interface. Recently, unconventional growth directions have also been observed for self-catalyzed nanowires. A small fraction of GaAsSb nanowires was found to grow in $\langle 112\rangle$ direction, ${ }^{17} \mathrm{GaAs}$ nanowires in the $\langle 111\rangle \mathrm{A}$ direction have been engineered, ${ }^{18}$ and InAs nanowires turning from $\langle 111\rangle \mathrm{B}$ to $\langle 11 \overline{2}\rangle$ direction have been demonstrated. ${ }^{19,20}$ However, tilted nanowires are not in all cases related to the growth in nonconventional crystalline directions. In some cases they can also explained by nucleation from parasitic growth (no crystalline relation with the substrate) or by multiple-order three-dimensional twinning. ${ }^{21} 3 \mathrm{D}$ twinning is based on the formation of other $\{111\}$ facets in additional to the (111)B growth front. A rotational twin around a nonvertical $\langle 111\rangle$ direction then allows formation of a secondary seed crystal, for which the $\langle 111\rangle \mathrm{B}$ directions are oriented differently compared to the original seed crystal. A multiple-order twinning process can therefore result in nanowires with a variety of quantized orientations, while the growth always proceeds by the formation of $\{111\} \mathrm{B}$ planes. $3 \mathrm{D}$ twinning was found in selfcatalyzed GaAs nanowires on silicon, ${ }^{21}$ as well as for goldcatalyzed InP nanowires. ${ }^{22}$ In the case of GaAs nanowires, further work has explained how multiple order twinning depends on the substrate preparation, ${ }^{23}$ and it has been shown that the wetting properties of the initial gallium droplets can be essential. $^{24}$ In the case of InAs nanowires grown without a foreign catalyst, tilted nanowires have been observed. ${ }^{25,26}$ However, the exact orientation has never been determined. Understanding the underlying mechanism of the formation of tilted InAs nanowires is particularly interesting, since the growth mechanism of MBE grown InAs nanowires is still a matter of debate, and both $\mathrm{VLS}^{26}$ and vapor-solid (VS) growth $^{27,28}$ have been reported under different conditions. The

Received: April 5, 2017

Revised: $\quad$ May 6, 2017

Published: May 9, 2017 
observation of multiple-order twinning would suggest that the growth is initiated with an indium droplet. The existence of tilted nanowires could therefore be an indicator of vaporliquid-solid growth as opposed to vapor-solid growth.

In this work, we provide a quantitative analysis and crystallography of tilted InAs nanowires grown by $\mathrm{MBE}$ without a foreign catalyst. We find that tilted nanowires occur at quantized angles, and show that most of these angles match those which are theoretically expected from a 3D twinning process. In addition, InAs nanowires oriented in $\langle 112\rangle$ and other low-index crystalline directions are demonstrated for the first time in the case of $\mathrm{MBE}$ growth without a foreign catalyst. By showing how the existence of tilted nanowires depends on the growth conditions we offer an approach to increase the number of tilted nanowires or suppress their formation completely-whichever is desired for a specific application.

\section{RESULTS AND DISCUSSION}

InAs nanowires are grown by molecular beam epitaxy (MBE) on GaAs(111)B substrates covered with $4.5 \mathrm{~nm}$ of HSQ oxide, as explained in ref 19. Figure $1 \mathrm{a}, \mathrm{b}$ shows representative top

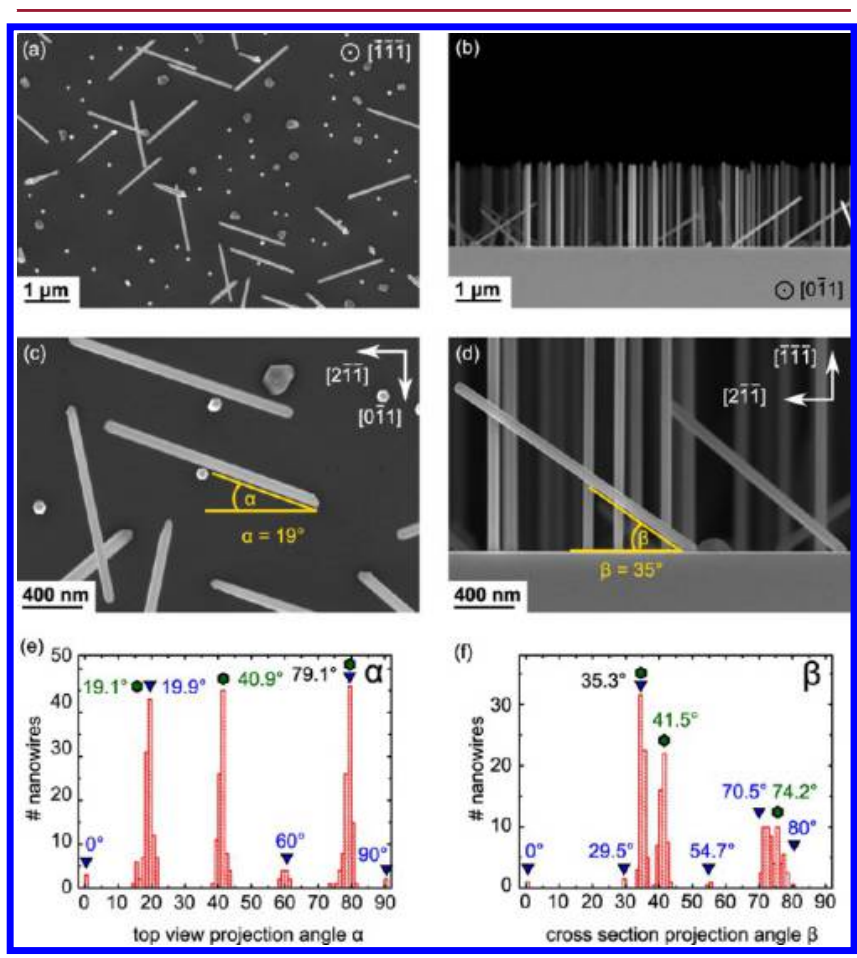

Figure 1. (a,b) Top view and cross-sectional SEM micrographs of a standard InAs nanowire sample. (c,d) Definition of the top view projection angle $\alpha$ and cross-sectional projection angle $\beta$. (e,f) Histograms of the occurrence of top view and cross-sectional projection angles. Green hexagons: 3D twinned wires. Blue triangles: $\langle 100\rangle,\langle 110\rangle,\langle 112\rangle$, or $\langle 111\rangle \mathrm{A}$ wires.

view and cross-sectional scanning electron micrographs (SEM) of samples obtained under typical conditions $\left(530{ }^{\circ} \mathrm{C}, \mathrm{V} / \mathrm{III}\right.$ flux ratio of 13.5). We clearly observe not only nanowires growing perpendicular to the substrate but also some tilted nanowires. In order to determine the orientation of the nanowires we analyze their alignment with respect to main crystallographic directions of the substrate, both in top-view and cross-sectional SEM images. In Figure 1c we define the top view angle $\alpha$ as the angle between the nanowire projection onto the (111) plane and the $[\overline{2} 11]$ direction (or the equivalent

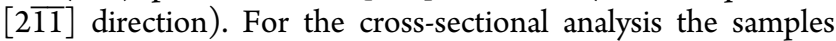
were cleaved along the $(0 \overline{1} 1)$ plane. We define the crosssectional angle $\beta$ as the angle between the projection of the nanowire onto the $(0 \overline{1} 1)$ plane and the $[\overline{2} 11]$ or $[2 \overline{11}]$ direction as shown in Figure 1d. Top view and cross-sectional projection angles were measured on several hundred nanowires in samples obtained with a variety of growth conditions (V/III ratios of 4.3-25; growth temperatures of $\left.490-550{ }^{\circ} \mathrm{C}\right)$. The resulting histograms are shown in Figure 1e,f for the top view and cross-sectional angles, respectively. For the top view projection angle, we observe that tilted nanowires occur predominantly at $\alpha \approx 19^{\circ}, 41^{\circ}$, and $79^{\circ}$. Cross-sectional angles show populations at $\beta \approx 35^{\circ}, 41^{\circ}$, and $70-80^{\circ}$. These measurements demonstrate that the tilted nanowires do not grow in random orientations and confirm the existence of a crystallographic relation with the substrate. The calculated projection angles which are marked in Figure 1e,f are explained in the following paragraph.

To analyze the orientation of the tilted nanowires, we calculate the projection angles of all low index crystalline directions $(\langle 100\rangle,\langle 110\rangle,\langle 111\rangle \mathrm{A},\langle 112\rangle)$, as well as the expected angles for nanowires after $3 \mathrm{D}$ twinning. The projection angles can be calculated by first calculating the projection of the wire onto a projection plane

$$
\overrightarrow{\text { proj }}=\overrightarrow{\text { wire }}-\frac{\overrightarrow{\text { wire }} \cdot \overrightarrow{\text { plane }}}{\left.\right|_{\text {plane }} ^{2}} \overrightarrow{\text { plane }}
$$

where $\overrightarrow{\text { wire }}$ is the vector corresponding to the nanowire growth direction, and $\overrightarrow{\text { proj }}$ is the projection of the nanowire onto the projection plane which is defined by its normal vector $\overrightarrow{\text { plane. }}$. Then the angle $\alpha$ with respect to the reference direction $\overrightarrow{\text { ref }}$ can be calculated by

$$
\cos (\alpha)=\frac{\overrightarrow{\mathrm{ref}} \cdot \overrightarrow{\mathrm{proj}}}{\overrightarrow{\mid \mathrm{ref}}|| \overrightarrow{\mathrm{proj}} \mid}
$$

For the top view projection angle we choose $\overrightarrow{\text { plane }}$ as $[111]$ and $\overrightarrow{\mathrm{ref}}$ as $[\overline{2} 11]$ or $[2 \overline{11}]$, while for the cross-sectional angle we choose $\overrightarrow{\text { plane }}$ as $[0 \overline{1} 1]$. For the calculation of nanowire directions after multiple-order twinning we assume the formation of a seed crystal with a $(\overline{111})$ top facet since the substrate is a $\operatorname{GaAs}(111) \mathrm{B}$ wafer. While growth then predominately occurs in the $[\overline{111}]$ direction with $\{1 \overline{1} 0\}$ facets leading to the hexagonal shape of nanowires, the seed crystal can also form additional facets in other $\langle 111\rangle$ directions. Twinning can now also occur around those $\langle 111\rangle$ directions and is referred to as $3 \mathrm{D}$ twinning. The crystalline directions of the $3 \mathrm{D}$ twinned crystal $\overrightarrow{x_{\text {rot }}}$ can therefore be calculated by

$$
\vec{x}_{\text {rot }}=R \cdot \vec{x}
$$

where $\vec{x}$ is the original direction and $R$ is the rotation matrix around the direction $\vec{u}=\left(u_{1}, u_{2}, u_{3}\right)$ with $u_{1}^{2}+u_{2}^{2}+u_{3}^{2}=1$. For a twin, $\vec{u}$ is the normalized vector of one of the $\langle 111\rangle$ directions, and is $R$ is given by a $180^{\circ}$ rotation; therefore 


$$
R=\left[\begin{array}{ccc}
-1+2 u_{1}^{2} & 2 u_{1} u_{2} & 2 u_{1} u_{3} \\
2 u_{1} u_{2} & -1+2 u_{2}^{2} & 2 u_{2} u_{3} \\
2 u_{1} u_{3} & 2 u_{2} u_{3} & -1+2 u_{3}^{2}
\end{array}\right]
$$

The coordinate system in our calculations corresponds to the orientation of the original seed crystal (and therefore the substrate). The rotated directions are obtained as a vector in this coordinate system and can as a consequence have higher indices. For clarity we denote the directions (and families of directions) before $3 \mathrm{D}$ twinning as $\left[x_{1} x_{2} x_{3}\right]$ (and $\left\langle x_{1} x_{2} x_{3}\right\rangle$ ), while the rotated directions are given as a vector $\left(x_{1}, x_{2}, x_{3}\right)$. One should note that the directions of the rotated crystal are not in agreement with its crystalline orientations anymore.

Using this approach we calculate the orientations of the four $\langle 111\rangle \mathrm{B}$ directions after twinning around the other three $\langle 111\rangle$ directions. The resulting 12 first-order 3D twinned orientations are all found to be in equivalent $(1,1,5)$ directions. Figure $2 \mathrm{a}$

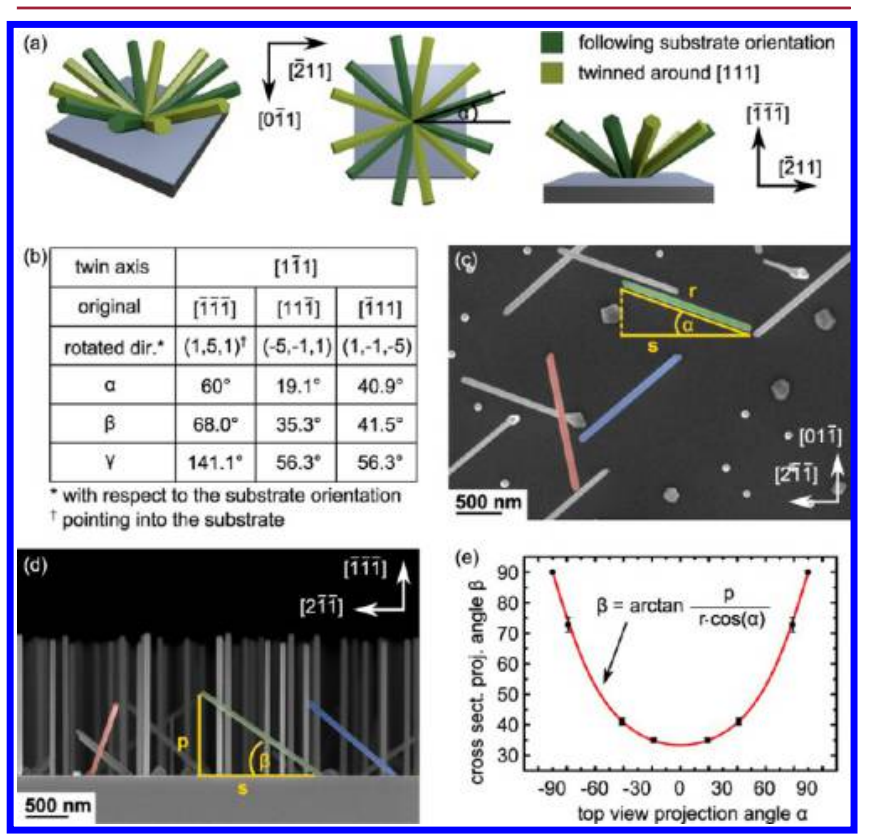

Figure 2. (a) 3D model of nanowire directions after first order 3D twinning. (b) Table summarizing the expected angles and directions of three new $\langle 111\rangle \mathrm{B}$ directions after twinning around the [1111] direction. (c,d) Top view and cross-sectional SEM micrographs with wires in equivalent orientations color-coded, and projected lengths and angles annotated. (e) Plot of $\beta$ as a function of $\alpha$ and a fit of their empirical relation.

shows a 3D model of nanowires growing in these new $\langle 111\rangle \mathrm{B}$ directions (only nanowires directed out of the substrate are shown). Since the original seed crystal can also be twinned with respect to the substrate (a twin around $[\overline{111}]$ ), we obtain six more directions. The two families of orientations are shown in dark and light green, respectively. We also present a top view projection and a cross-sectional projection of the model. The table shown in Figure $2 \mathrm{~b}$ gives the expected directions and angles for new $\langle 111\rangle \mathrm{B}$ directions after twinning around the [11ㅣ direction. In addition to the projection angles $\alpha$ and $\beta$ we also report $\gamma$, which corresponds to the angle between [111] and the new $\langle 111\rangle \mathrm{B}$ directions. We observe that one of the new directions is pointing into the substrate (denoted by $\dagger$ ). The projection angles of the two out-of-plane directions $\alpha=19.1^{\circ}$, $40.9^{\circ}$, and $\beta=35.3^{\circ}, 41.5^{\circ}$ are in good agreement with peaks observed in the histograms presented in Figure 1e,f. The other angles observed in the histograms can be explained by calculating the twinning around the other $\langle 111\rangle \mathrm{B}$ directions, or other low index crystalline orientations (a complete table of the expected projection angles is presented in the Supporting Information). The observation that the most pronounced peaks in the histogram correspond to angles which are expected after first order $3 \mathrm{D}$ twinning are a strong indication for the origin of tilted nanowires, but it is not enough as a proof since top view and cross-sectional projection angles are never measured on the same nanowire. Furthermore, as denoted in the histogram, several projection angles can result from both $3 \mathrm{D}$ twinned nanowires as well as other low index crystalline orientations (e.g., $\beta=35.3^{\circ}$ is expected for $\langle 100\rangle,\langle 111\rangle \mathrm{A},\langle 112\rangle$, and 3D twinned nanowires). To gain more insight, we propose a way to calculate the real angle $\gamma$ of the nanowire direction with respect to the surface normal ([$\overline{111}]$ direction), by matching top view angles $\alpha$ with cross-sectional angles $\beta$. This approach is based on the observation that all $3 \mathrm{D}$ twinned orientations exhibit the same real angle $\gamma=56.3^{\circ}$, while other low index orientations exhibit different angles. We can then use that for nanowires with the same angle $\gamma$; a small top view angle $\alpha$ corresponds a small cross-sectional angle $\beta$ (and vice versa). Therefore, nanowires from top view images can be matched with nanowires from cross-sectional images. Figure $2 \mathrm{c}, \mathrm{d}$ shows SEM micrographs of nanowires which are color-coded accordingly. From this figure we also derive an empirical relation between $\alpha$ and $\beta$ as follows: In Figure 2c we define $r$ to be the length of the projection of the nanowire onto the (111) plane, and $s$ as the component of $r$ along the [211] or [2 $\overline{11}]$ direction. From this follows $s=r \cdot \cos \alpha$. In Figure $2 \mathrm{~d}$ we see a projection of the nanowire onto the $(0 \overline{1} 1)$ plane, and define $p$ as the component of this projection along the $[\overline{111}]$ direction, again finding $s$ as the component along the $[\overline{2} 11]$ or $[2 \overline{11}]$ direction. Taking $\beta=\arctan \left(\frac{p}{s}\right)$ and combining it with $s=r$. $\cos \alpha$ we find the empirical relation between $\beta$ and $\alpha$ as

$$
\beta=\arctan \left(\frac{p}{r \cdot \cos \alpha}\right)
$$

From the histograms, we now match the three predominant peaks of top view angles $\alpha$ with cross-sectional angles $\beta$. Figure $2 \mathrm{e}$ shows a plot of the matched angles, including two theoretical points at $\beta=90^{\circ}$ for $\alpha= \pm 90^{\circ}$. The data points are fitted with eq 5 giving $p / r=0.66 \pm 0.01$. The accuracy of the fit indicates that the predominant projection angles result from a family of orientations which exhibit the same angle $\gamma$ (and thereby validates our direct matching approach). At $\alpha=0^{\circ}$ we find $\gamma=90^{\circ}-\beta=90^{\circ}-\arctan (p / r)=55.6^{\circ} \pm 0.6^{\circ}$, which is in agreement with the calculated angle of $56.3^{\circ}$ for all first order twin directions. We have thus comprehensively shown that the predominant projection angles of tilted nanowires match those expected from first order 3D twinning.

We now turn to the analysis of the nanowire crystal structure of tilted nanowires by transmission electron microscopy (TEM). Our focus lies on the foot of the nanowires, where 3D twinning is expected to occur. For the analysis, wires from a sample showing a significant density of tilted nanowires were broken off of the substrate and transferred to TEM grids by sweeping the sample with a grid. Figure 3 a shows crosssectional and top view SEM micrographs of a sample before and after transferring the nanowires. From the cross-sectional 


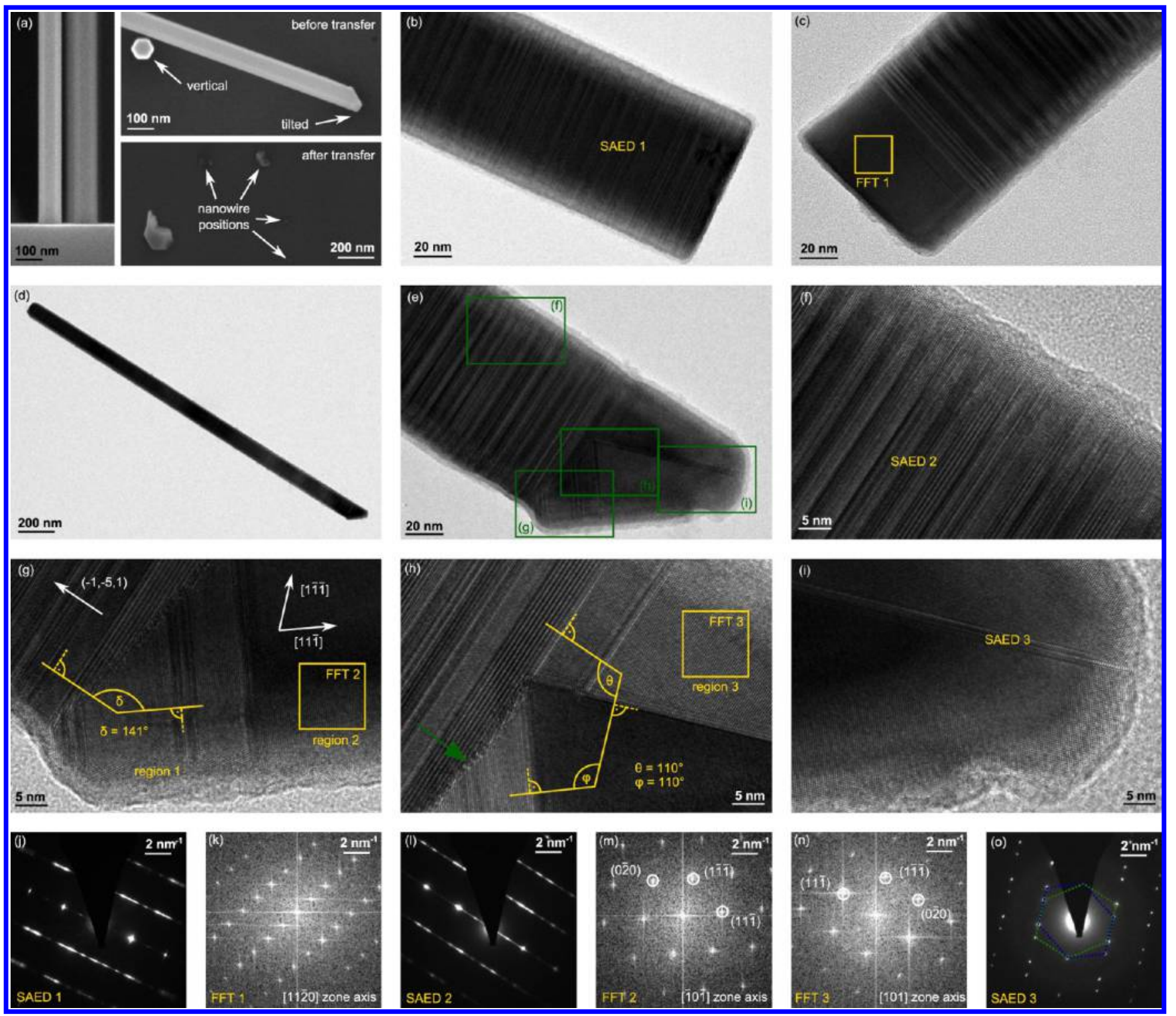

Figure 3. (a) Left: cross-sectional SEM micrograph of vertical nanowires. Right: top view SEM micrographs of a sample before and after transferring the nanowires to a TEM grid. (b,c) TEM micrographs of the foot of two vertical nanowires. (d) Overview TEM micrograph of a nanowire showing a similar foot as observed for the tilted nanowires by SEM. (e) Magnified image of the nanowire foot. (f-i) High-resolution TEM micrographs of the polytypic nanowire, the bottom left part of the foot, the center, and the bottom right part. $(j-o)$ Selected area electron diffraction pattern and FFT images of the regions indicated.

image we observe that the foot of vertical nanowires is completely straight, while the top view image before nanowire transfer shows that the foot of tilted nanowires exhibits facets at a particular angle. After transferring the nanowires, the sample appears almost empty, with small crystallites remaining from the nanowires (indicated by arrows). This shows that most nanowires break off directly from the base, and the specific shape of the nanowire foot can therefore be used to distinguish tilted from vertical nanowires on the TEM grid. Figure $3 b, c$ shows TEM micrographs of the foot of two different nanowires. We observe no change in morphology along the nanowire length. For the first sample, the crystal structure shows perpendicular stacking faults all along the nanowire. The polytypic crystal structure can also be observed in the selected area electron diffraction (SAED) pattern shown in Figure 3j. The second nanowire does not show any change in morphology either, and the crystal structure also shows stacking defects perpendicular to the growth direction. At the very beginning of the nanowire, a short Wurtzite (WZ) section can be observed, as shown by the fast Fourier transform (FFT) in Figure 3k. More information about the standard nanowire crystal structure of vertical nanowires can be found in the Supporting Information. Figure 3d shows a low-magnification TEM micrograph of a nanowire which exhibits a nanowire foot that looks similar to the ones observed in SEM images of tilted nanowires. A magnified TEM micrograph of the nanowire foot is presented in Figure 3e, with a change in crystal structure at the very beginning of the growth. Figure $3 f-i$ shows highresolution TEM micrographs of different locations of the nanowire foot (as indicated in (e)). In Figure $3 \mathrm{f}$ we observe the standard polytypic crystal structure with stacking defects perpendicular to the growth direction, which is also confirmed by the selected area diffraction pattern in Figure 3l. Figure $3 g$ shows a high-resolution TEM micrograph of the left corner of 
the nanowire foot. We observe a zincblende (ZB) region with a few stacking defects on the left and then a pure zincblende region. An FFT image of the pure zincblende region is shown in Figure $3 \mathrm{~m}$ and can be indexed, assuming that the image was taken in the $[\overline{1} 0 \overline{1}]$ zone axis. The angle between the $[11 \overline{1}]$ direction of the pure zincblende region and the nanowire growth direction is found to be $141^{\circ}$. Figure $3 \mathrm{~h}$ shows a highresolution TEM micrograph of the center of the nanowire foot. The FFT of region 3 is shown in Figure $3 \mathrm{n}$ and can be indexed to show the crystal from the [101] zone axis. The angles between region 2 , region 3 , and the nanowire growth direction are found to be $110^{\circ}$, which is in agreement with the angle expected between two $\langle 111\rangle$ directions. Figure $3 i$ shows the bottom right part of the nanowire foot. The corresponding selected area diffraction image in Figure 30 confirms the observation of the two zincblende regions with one rotational twin. In Figure $3 \mathrm{~h}$ one can also observe that the interface between the bottom right part of the foot and the polytypic nanowire is perfectly crystalline, while the interface between the bottom left part and the polytypic nanowire (indicated with a green arrow) is defective, indicating that two different seed crystals merged here. Combining the information from the different micrographs and FFTs images, we suggest that growth started in region 2 , followed by a twin around the $[1 \overline{11}] \mathrm{A}$ direction to form region 3 . Region 3 then started to exhibit a growth front in a new $\langle 111\rangle \mathrm{B}$ direction, which then continued to grow as a nanowire. The growth front of the elongated nanowire corresponds to the $[11 \overline{1}]$ direction in the coordinate system of region 3 . Converting this direction to the original coordinate system of region 2 results in the vector $(-1,-5,1)$, which indeed forms an angle of $141^{\circ}$ with the [11 $\left.\overline{1}\right]$ direction of region 2. The bottom left part of the nanowire foot (region 1) then accumulated material until the gap was filled, explaining the defective interface with the final nanowire. We conclude that even though an unambiguous identification of the crystalline directions of a nanowire that has been removed from the substrate is not possible, a multiorder twinning process can explain the change in crystalline direction which is observed experimentally. More TEM images of other nanowire feet which show stacking defects nonperpendicular to the growth direction can be found in the Supporting Information.

We continue by exploring how the existence of tilted nanowires depends on the growth conditions. Figure $4 a-e$ shows top view and cross-sectional SEM micrographs of samples that were grown under a V/III flux ratio of 4.3, 7.1, $13.5,17.9$, and 25 , respectively. For this, the arsenic beam equivalent pressure (BEP) was changed as indicated in the figure, while all other growth conditions were kept constant. Figure $4 \mathrm{a}$ shows a high density of tilted nanowires for the lowest V/III ratio. A slightly lower density can be observed in Figure $4 \mathrm{~b}$. The sample in Figure $4 \mathrm{c}$ shows only very few tilted nanowires, and the two samples presented in Figure 4d,e show no tilted nanowires. To quantify our finding we counted the density of tilted nanowires and vertical nanowires. The percentage of tilted nanowires defined as $p=$ tilted $/$ (tilted + vertical) is presented in Figure 4f, showing a dramatic decrease of tilted nanowires with increasing V/III ratio. For each V/III ratio, several SEM micrographs with hundreds of nanowires were taken into account. The data represents the average and standard deviation of the nanowire density on different SEM images. The statistics also include a sample grown at a V/III ratio of 15 , for which SEM micrographs will be presented as reference sample in Figure 6. One should note that with

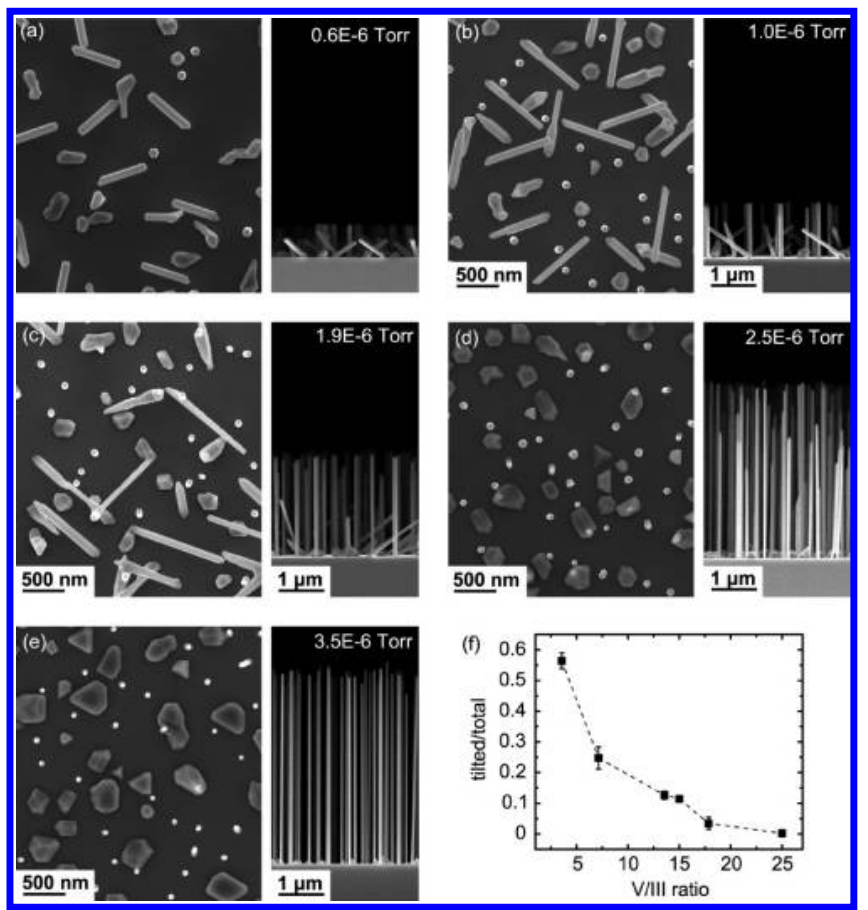

Figure 4. Arsenic dependence of the existence of tilted wires. (a-e) Top view and cross-sectional SEM micrographs of samples grown at a V/III ratio of 4.3, 7.1, 13.5, 17.9, and 25, respectively. (f) Percentage of tilted nanowires.

increasing $\mathrm{V} / \mathrm{III}$ ratio also the optimal growth temperature slightly increases. For this study all samples were grown at a temperature of $530{ }^{\circ} \mathrm{C}$ and the analysis was done on the part of the wafer which showed the best nanowire homogeneity (i.e., the center for samples $(\mathrm{a}-\mathrm{c})$ and the edge for samples $(\mathrm{d}, \mathrm{e}))$. The change in nanowire morphology across the sample is related to a slight temperature gradient $\left(\sim 10{ }^{\circ} \mathrm{C}\right)$ due to the manipulator design in our MBE. The decrease in tilted nanowires reported here, however, is not related to the temperature; a higher growth temperature in fact favors the formation of tilted nanowires, as discussed in the Supporting Information. A strong dependence of the formation of tilted nanowires on the V/III ratio is in agreement with results observed in the literature, where nanowires are found to be all vertical for samples grown at high V/III ratio of 120,200 , or 320 (refs 28-30.), while some tilted nanowires are observed at a V/III ratio of 7-8 (ref 26.). One should note that the effective V/III ratio also depends on the growth temperature (due to re-evaporation from the substrate), explaining why no tilted nanowires are observed at a V/III ratio of 6.3 and a significantly lower growth temperature. ${ }^{27}$ The V/III ratio dependence of the existence of tilted nanowires could be explained considering indium droplets as seed particles: A higher arsenic pressure leads to smaller indium seed droplets at the early stages of growth (or a complete absence of droplets), therefore avoiding the formation of additional $\{111\}$ facets leading to a change of growth direction. This explanation implies that a catalyst droplet is present at the beginning of growth under low V/III ratio where tilted nanowires are observed, and absent (or significantly smaller) for nanowires grown at higher V/III ratios. Additionally, the existence of an elongated WZ section at the foot of nanowires grown at high $\mathrm{V} / \mathrm{III}$ ratios (ref 25) may suppress $3 \mathrm{D}$ twinning, since the 


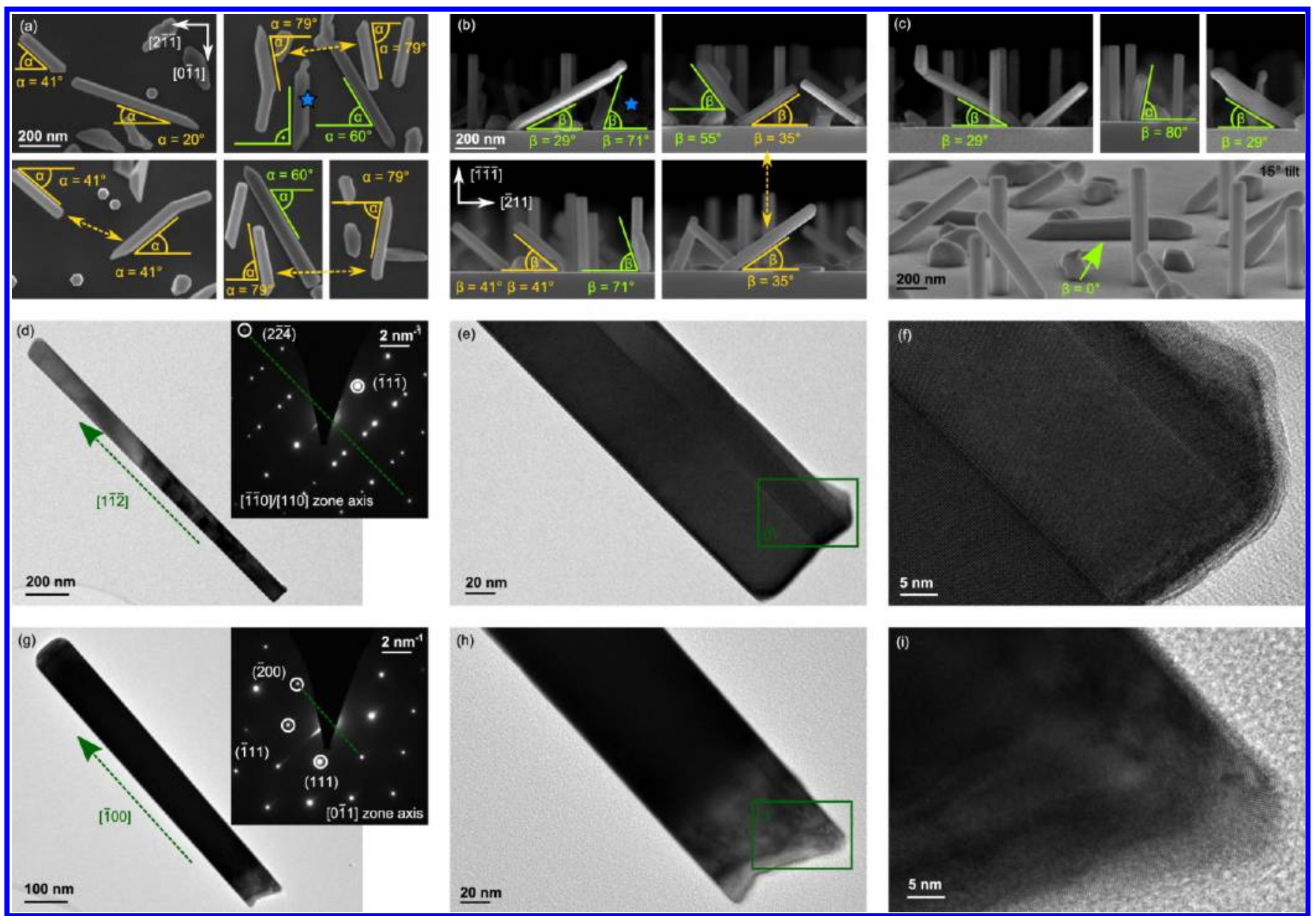

Figure 5. Detailed SEM and TEM analysis of a sample grown at a V/III ratio of 4.3. (a-c) Top view and cross-sectional SEM micrographs. Projection angles that can be explained by multiorder twinning are marked in yellow and other angles are marked in green. (d-f) TEM micrographs and SAED pattern of a nanowire growing in $[1 \overline{12}]$ direction. $(\mathrm{g}-\mathrm{i})$ TEM micrographs and SAED pattern of a nanowire growing in [100] direction.

formation of new $\langle 111\rangle \mathrm{B}$ facets would be difficult in a hexagonal WZ seed crystal.

At very low $\mathrm{V} / \mathrm{III}$ ratio we also observe projection angles that cannot be explained by multiple-order twinning. Figure 5 shows a more detailed SEM and TEM analysis of the sample presented in Figure 4a, which was grown at a V/III ratio of 4.3 (arsenic pressure of $6 \times 10^{-7}$ Torr). Top-view SEM micrographs are presented in Figure 5a. We observe top-view projection angles of approximately $20^{\circ}, 41^{\circ}, 60^{\circ}, 79^{\circ}$, and $90^{\circ}$. Projection angles of $60^{\circ}$ and $90^{\circ}$ (highlighted in green) cannot be explained by multiple-order twinning (see table with all projection angles of low-index crystalline orientations in Supporting Information). Orientations corresponding a projection angle of $60^{\circ}$ and $90^{\circ}$ could be in fact $\langle 100\rangle,\langle 110\rangle,\langle 112\rangle$, and $\langle 111\rangle \mathrm{A}$ growth directions. One should note that the calculation of projection angles also includes certain $\langle 110\rangle$ and $\langle 112\rangle$ directions which are found to be parallel to the substrate (crawling nanowires). Unfortunately, analyzing only top view angles is not conclusive, since many wire orientations show similar top view angles. In particular, the top view projection angles of $\sim 20^{\circ}, 41^{\circ}$, and $79^{\circ}$ can result from both 3D twinned nanowires as well as $\langle 112\rangle$ nanowires. In Figure 5a we highlighted a few nanowires that have the same projection angle but different morphologies/facets with arrows. This can be explained by different growth directions: while $3 \mathrm{D}$ twinned nanowires have a hexagonal shape with six $\{110\}$ facets, $\langle 112\rangle$ oriented nanowires are expected to have rectangular shape with two $\{110\}$ and two $\{111\}$ facets. Figure $5 b, c$ shows crosssectional SEM micrographs of the same sample. Here we observe projection angles of $0^{\circ}, 29^{\circ}, 35^{\circ}, 41^{\circ}, 55^{\circ}, 71^{\circ}$, and $80^{\circ}$, where $0^{\circ}$ corresponds to crawling wires as shown in the $15^{\circ}$ tilted image. Comparing with the table of projection angles we find that (i) $35^{\circ}$ and $41^{\circ}$ can be explained by $3 \mathrm{D}$ twinning; (ii) $29^{\circ}, 35^{\circ}, 71^{\circ}$, and $80^{\circ}$ would be consistent with $\langle 112\rangle$ oriented nanowires; and (iii) $55^{\circ}$ and $71^{\circ}$ would agree with the projection of $\langle 110\rangle$ oriented nanowires. The angles $29^{\circ}, 55^{\circ}$, $71^{\circ}$, and $80^{\circ}$ which are exclusive to $\langle 110\rangle$ and $\langle 112\rangle$ orientations are highlighted in green. Again, we also highlighted two nanowires with the same projection angle but clearly different faceting with an arrow. We also remark that a crosssectional projection angle of $19^{\circ}$ is not observed in any sample, indicating that $\langle\overline{11} 1\rangle \mathrm{A}$ oriented nanowires are very rare or not present at all. This observation stands in contrast to the case of GaAs nanowires grown at very low V/III ratio, where a significant increase of A-polar wires was reported. ${ }^{18}$ Figure $5 \mathrm{~d}-\mathrm{i}$ presents TEM micrographs of nanowires with unusual growth directions. Figure $5 \mathrm{~d}$ shows a low resolution image and SAED pattern of the first nanowire. The SAED diffraction pattern shows zincblende stacking and two twin orientations which can be indexed assuming a [110] or [110] zone axis. By comparing the growth direction of the nanowire with the diffraction pattern, it can be observed that the nanowire axis corresponds 

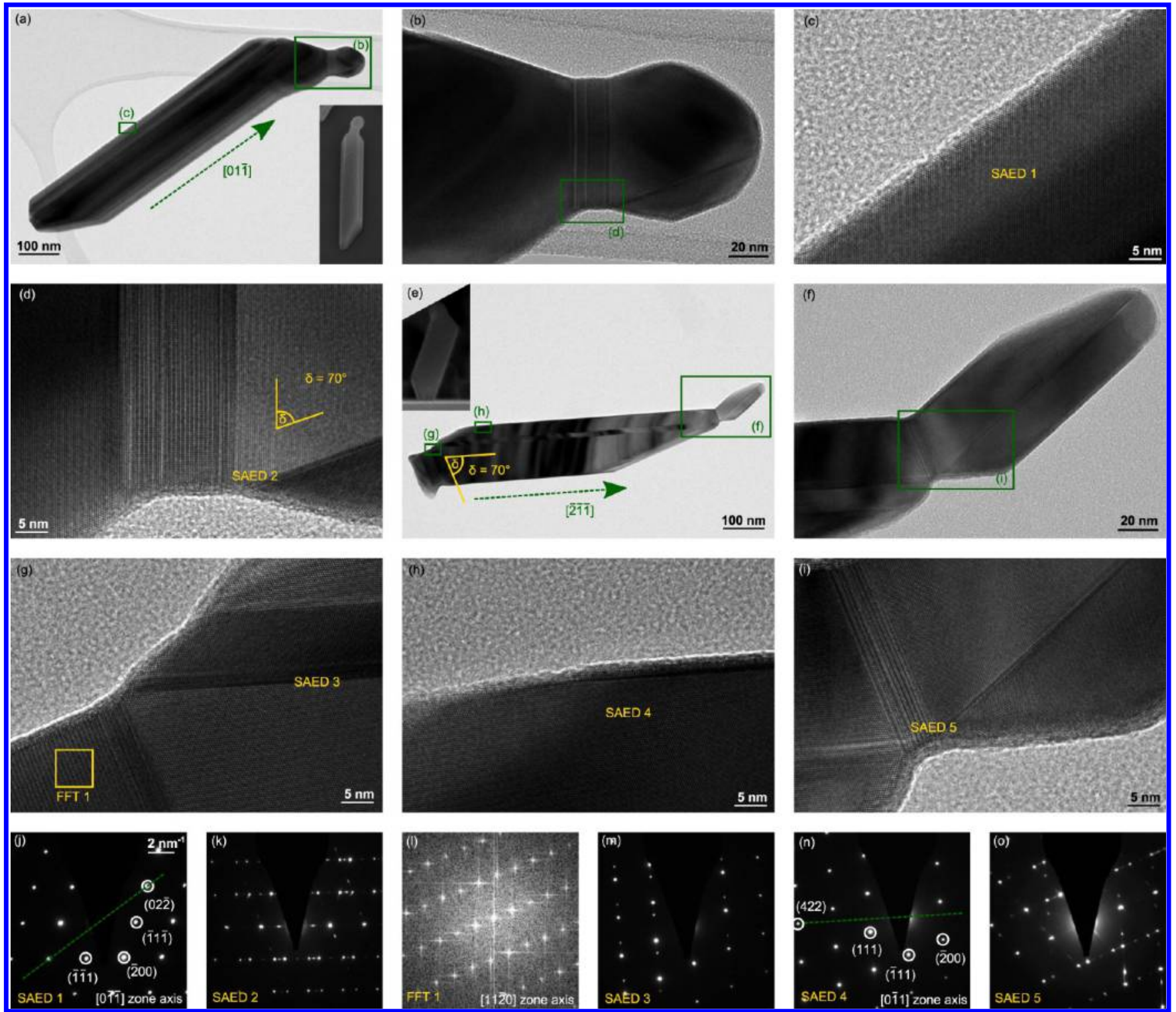

Figure 6. TEM analysis of nanowires with unusual morphology. $(a-d)$ TEM micrographs of a nanostructure growing in $[01 \overline{1}]$ direction. Parts (j,k) present the corresponding diffraction images. $(\mathrm{e}-\mathrm{i})$ TEM micrographs of a nanostructure growing in the [211] direction. The corresponding diffraction images are shown in $(j-0)$.

to the $[1 \overline{12}]$ direction. Figure 5e,f shows a magnified image of the nanowire foot and a high-resolution TEM micrograph of the region indicated. The images show zincblende stacking with two rotation twins running along the nanowire (parallel to the growth axis). These longitudinal defects are characteristic for nanowires in the $\langle 112\rangle$ direction. ${ }^{16,31}$ TEM analysis of two more $\langle 112\rangle$ oriented nanowires are presented in the Supporting Information. Figure $5 \mathrm{~g}$ shows a low resolution TEM micrograph and SAED pattern of another nanowire. The diffraction pattern can be indexed assuming a $[0 \overline{1} 1]$ zone axis and the nanowire growth direction therefore corresponds to the [100] direction. The expected rectangular shape of $\langle 100\rangle$ nanowires explains why the nanowire appears very dark and no thinner region at the edges can be found. Figure 5h,i shows a magnified image and a high-resolution TEM micrograph of the foot of the nanowire, and confirms a defect-free zincblende crystal structure.

As seen from the SEM micrographs in Figure 5, nanowires in unusual orientations often show kinks and other changes in morphology. Figure 6 presents TEM micrographs of two nanowires showing an unusual morphology. Figure 6a shows a low magnification TEM micrograph of a nanowire resembling the morphology of the nanowire oriented in the [01 $\overline{1}]$ direction, which is marked with a star in the SEM image in Figure 5a. We observe a long defect-free part and then a change of direction and a small droplet. A magnified image of the tip region is shown in Figure $6 \mathrm{~b}$, showing a few defects at the interface. Figure $6 c, d$ shows high-resolution TEM images of the defect-free nanowire and the defects at the tip. The SAED image of the defect-free region is presented in $(j)$ and shows a pure $\mathrm{ZB}$ crystal structure which can be indexed assuming a $[0 \overline{11}]$ zone axis. We also present dashed lines corresponding to the direction of the nanowire both in the TEM micrograph and in the SAED image. From the SAED we observe that the nanowire direction is $[01 \overline{1}]$, which matches the direction observed in the SEM micrograph (it should be noted that this corresponds to a crawling nanowire). The defects near the tip can be identified as a few layers of WZ, and then the droplet 
itself shows a twin defect in a $\langle 111\rangle$ direction at a $70^{\circ}$ angle with respect to the $\mathrm{WZ}$ layers. The existence of the two polytypes and the rotational twin is confirmed by the SAED image shown in (k). Figure 6e shows a low magnification TEM micrograph of another nanowire, which resembles the small nanowire marked with a star in the background of the SEM image of Figure 5b. We observe a few defects at the foot of the nanowire and then an elongated section showing two defects parallel to the growth direction, and then a tip in a different direction. A magnified image of the tip region is presented in Figure 6 f. Figure $6 \mathrm{~g}-\mathrm{i}$ shows high resolution TEM micrographs of the foot of the wire, the main part, and the transition to the tip. Figure $6 \mathrm{~g}$ shows the foot of the nanowire and the beginning of the main part. We observe a few layers of WZ at the foot of the nanowire, which can also be seen in the FFT image in (1). The beginning of the main nanowire core shows $\mathrm{ZB}$ crystal structure with two twin defects, which can also be seen in the SAED image in $(\mathrm{m})$. The main core of the nanowire is $\mathrm{ZB}$ as shown in (h) and the corresponding SAED shown in ( $\mathrm{n}$ ) can be indexed assuming a $[0 \overline{1} 1]$ zone axis. The dashed green line corresponds to the growth direction of the nanowire and can be found to be oriented in the $[\overline{211}]$ direction. The transition to the tip (Figure 6i) shows again a few layers of WZ stacking and then a $Z B$ region with a twin defect in the $\langle 111\rangle$ direction at an angle of $70^{\circ}$ with respect to the WZ layers. Figure 60 shows a SAED image of the whole tip region, showing diffraction spots from WZ stacking (very weak) as well as the $\mathrm{ZB}$ sections showing twins in two different directions. Summarizing the analysis in Figure 6, we find projection angles that can only be explained by $\langle 110\rangle$ and $\langle 112\rangle$ oriented nanowires, and TEM images support the existence of nanowires in those unconventional directions.

We have suggested the existence of the droplet at the beginning of growth as a possible explanation for formation of tilted nanowires. To study this hypothesis we investigate nanowire growth after the predeposition of indium droplets. For this, we first heat up and anneal a sample for $1 \mathrm{~h}$ at $480{ }^{\circ} \mathrm{C}$ under arsenic flux (as for standard samples). The arsenic valve is then closed and indium is deposited at the standard indium BEP of $1.4 \times 10^{-7}$ Torr. Finally the sample is heated to the growth temperature of $530{ }^{\circ} \mathrm{C}$ and nanowires are grown for $1 \mathrm{~h}$. As growth conditions we choose a relatively high V/III ratio of 15 . Figure 7 a shows a top view and cross-sectional SEM micrograph of a reference sample without indium predeposition, showing a very low density of tilted nanowires, as expected at high V/III ratio. Figure $7 \mathrm{~b}$ shows a sample where indium was deposited for $3 \mathrm{~min}$ and then nanowire growth was started under identical growth conditions. A higher density of tilted nanowires can be observed, while the density of vertical nanowires dramatically decreases. The green arrow highlights a nanocross which was formed by merging of two tilted nanowires. Figure $7 \mathrm{c}$ shows a sample with a $10 \mathrm{~min}$ indium predeposition. Almost no vertical nanowires are found and only very few tilted nanowires. Instead the sample is covered with parasitic growth. In the inset, a top view SEM micrograph of a reference sample is presented, for which the process was stopped after the $10 \mathrm{~min}$ indium predeposition. We observe that the sample is covered with droplets, confirming that indium droplets can be formed under the conditions studied. Statistics on the percentage of tilted nanowires and the overall density are shown in Figure $7 \mathrm{~d}$. Our results show that predeposition of indium dramatically increases the occurrence of tilted nanowires, even under growth conditions for which

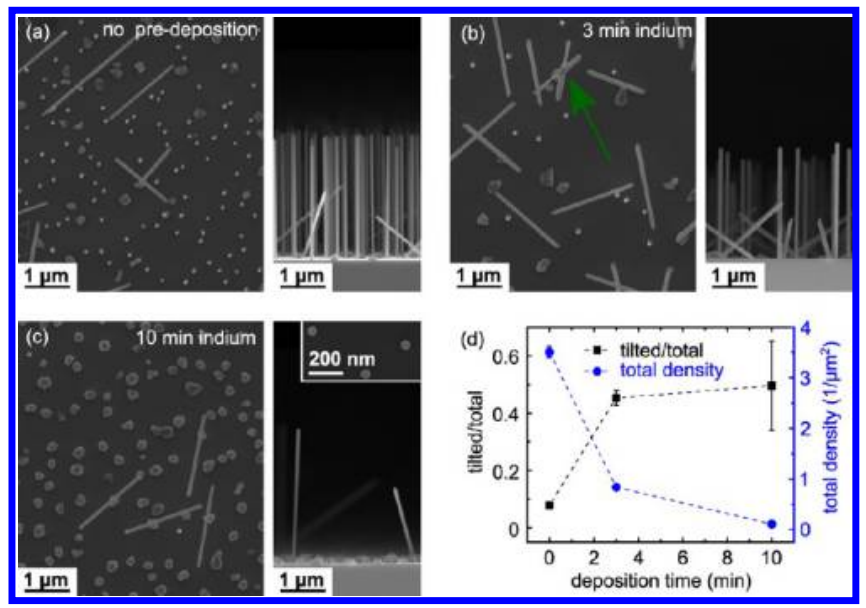

Figure 7. Nanowire samples grown at a V/III ratio of 15. (a) Top view and cross-sectional micrographs of a reference sample without indium deposition. (b,c) Samples with 3 and $10 \mathrm{~min}$ indium deposition, respectively. The inset in (c) shows a sample just after 10 min indium predeposition. (d) Statistics of the percentage of tilted nanowires and the nanowire density.

normally only very few tilted nanowires are observed. This finding supports our hypothesis that the formation of tilted nanowires is related to the existence of an indium droplet at the beginning of growth. We can therefore conclude that under low V/III ratio nanowire growth starts with a droplet, while under high V/III ratio the droplet is significantly smaller or absent completely.

Finally, we also study the existence of tilted nanowires as a function of the growth temperature and the oxide thickness. All SEM micrographs and statistics can be found in Supporting Information. We find that increasing the growth temperature increases the ratio of tilted nanowires, which can be attributed to a change in effective V/III ratio, as mentioned above. With respect to the oxide thickness we find that a thicker oxide favors the formation of tilted nanowires. This result can be compared to the work by Matteini et al., ${ }^{24}$ where a thicker native silicon oxide was found to favor spilling of gallium droplets and therefore nucleation of tilted nanowires. Whether or not the explanation of the surface energy also applies in our case is subject to further investigation. Last but not least, we also note that incorporation of antimony suppresses the formation of tilted nanowires, as can be observed from the antimony series in ref 25. Considering that incorporation of antimony dramatically reduces the formation of rotational twins, this finding further supports the explanation of tilted nanowires due to $3 \mathrm{D}$ twinning.

\section{CONCLUSION}

In conclusion we presented a complete analysis of the existence of tilted InAs nanowires obtained without a foreign catalyst. Tilted nanowires are found to occur at specific angles, which can in most cases be explained by a $3 \mathrm{D}$ twinning process. At very low V/III ratio, also angles corresponding to $\langle 112\rangle$ or other low-index orientations are found. TEM studies of both $3 \mathrm{D}$ twinned nanowires as well as nanowires growing in usual crystalline directions are presented. The formation of tilted nanowires depends on the growth conditions and on the substrate preparation. Most importantly we find that increasing the V/III ratio suppressed the formation of tilted nanowires almost completely. This result suggests that at low V/III ratios 
an indium droplet is present during the early stages of growth, allowing for the formation of different $\{111\}$ B facets, while at high V/III ratio multiorder twinning is suppressed, possibly due to the absence of a catalyst droplet. Our results contribute to the understanding of the growth mechanism of InAs nanowires, and provide several pathways to achieve all vertical nanowires-which is an important requirement for the fabrication of devices where several nanowires are contacted in parallel asgrown on the substrate. Tuning the ratio of tilted nanowires also constitutes a pathway to make nanowire junctions and crosses, which are important building blocks for Majorana Fermion braiding.

\section{ASSOCIATED CONTENT}

\section{S Supporting Information}

The Supporting Information is available free of charge on the ACS Publications website at DOI: 10.1021/acs.cgd.7b00487.

Detailed explanation of 3D twinning, a complete table of projection angles, additional TEM analysis, and the study of growth temperature and oxide thickness (PDF)

\section{AUTHOR INFORMATION}

\section{Corresponding Author}

*E-mail: anna.fontcuberta-morral@epfl.ch.

\section{ORCID $\odot$}

Anna Fontcuberta i Morral: 0000-0002-5070-2196

\section{Author Contributions}

H.P. and Y.v.H. grew the samples. Y.v.H. performed the quantitative analysis. H.P. performed the TEM analysis. H.P. and A.F.i.M designed the experiments. H.P., G.T., M.F., and J.B.L. calibrated and maintained the MBE system. H.P. made the figures and artwork. H.P., Y.v.H., and A.F.i.M. wrote and edited the manuscript. All authors discussed the results and commented on the manuscript.

Notes

The authors declare no competing financial interest.

\section{ACKNOWLEDGMENTS}

The authors acknowledge the SNF for financial support via the project 200021_169908 and through the NCCR QSIT.

\section{REFERENCES}

(1) Mourik, V.; Zuo, K.; Frolov, S. M.; Plissard, S. R.; Bakkers, E. P. a M.; Kouwenhoven, L. P. Signatures of Majorana Fermions in Hybrid Superconductor-Semiconductor Nanowire Devices. Science 2012, 336 (6084), 1003-1007.

(2) Das, A.; Ronen, Y.; Most, Y.; Oreg, Y.; Heiblum, M.; Shtrikman, H. Zero-Bias Peaks and Splitting in an Al-InAs Nanowire Topological Superconductor as a Signature of Majorana Fermions. Nat. Phvs. 2012, 8 (12), 887-895.

(3) Deng, M. T.; Yu, C. L.; Huang, G. Y.; Larsson, M.; Caroff, P.; Xu, H. Q. Anomalous Zero-Bias Conductance Peak in a Nb-InSb Nanowire-Nb Hybrid Device. Nano Lett. 2012, 12 (12), 6414-6419.

(4) Brongersma, M. L.; Cui, Y.; Fan, S. Light Management for Photovoltaics Using High-Index Nanostructures. Nat. Mater. 2014, 13 (5), 451-460.

(5) del Alamo, J. a. Nanometre-Scale Electronics with III-V Compound Semiconductors. Nature 2011, 479 (7373), 317-323.

(6) Li, Y.; Qian, F.; Xiang, J.; Lieber, C. M. Nanowire Electronic and Optoelectronic Devices. Mater. Todav 2006, 9 (10), 18-27.

(7) Takei, K.; Takahashi, T.; Ho, J. C.; Ko, H.; Gillies, A. G.; Leu, P. W.; Fearing, R. S.; Javey, A. Nanowire Active-Matrix Circuitry for LowVoltage Macroscale Artificial Skin. Nat. Mater. 2010, 9 (10), 821-826.
(8) Dayeh, S. a.; Aplin, D. P. R.; Zhou, X.; Yu, P. K. L.; Yu, E. T.; Wang, D. High Electron Mobility InAs Nanowire Field-Effect Transistors. Small 2007, 3 (2), 326-332.

(9) Plissard, S. R.; van Weperen, I.; Car, D.; Verheijen, M. a; Immink, G. W. G.; Kammhuber, J.; Cornelissen, L. J.; Szombati, D. B.; Geresdi, A.; Frolov, S. M.; Kouwenhoven, L. P.; Bakkers, E. P. a M. Formation and Electronic Properties of InSb Nanocrosses. Nat. Nanotechnol. 2013, 8 (11), 859-864.

(10) Car, D.; Wang, J.; Verheijen, M. A.; Bakkers, E. P. A. M.; Plissard, S. R. Rationally Designed Single-Crystalline Nanowire Networks. Adv. Mater. 2014, 26, 4875-4879.

(11) Kang, J.-H.; Galicka, M.; Kacman, P.; Shtrikman, H. Wurtzite/ Zinc-Blende "K"-Shape InAs Nanowires with Embedded TwoDimensional Wurtzite Plates. Nano Lett. 2017, 17 (1), 531-537.

(12) Fortuna, S. a; Li, X. Metal-Catalyzed Semiconductor Nanowires: A Review on the Control of Growth Directions. Semicond. Sci. Technol. 2010, 25 (2), 24005.

(13) Yuan, X.; Caroff, P.; Wong-Leung, J.; Fu, L.; Tan, H. H. Tunable Polarity in a III-V Nanowire by Droplet Wetting and Surface Energy Engineering. Adv. Mater. 2015, 27, 6096-6103.

(14) Zamani, R. R.; Gorji Ghalamestani, S.; Niu, J.; Sköld, N.; Dick, K. A. Polarity and Growth Directions in Sn-Seeded GaSb Nanowires. Nanoscale 2017, 9 (9), 3159-3168.

(15) Wang, J.; Plissard, S. R.; Verheijen, M. A.; Feiner, L. F.; Cavalli, A.; Bakkers, E. P. A. M. Reversible Switching of InP Nanowire Growth Direction by Catalyst Engineering. Nano Lett. 2013, 13 (8), 38023806.

(16) Li, Z. A.; Möller, C.; Migunov, V.; Spasova, M.; Farle, M.; Lysov, A.; Gutsche, C.; Regolin, I.; Prost, W.; Tegude, F. J.; Ercius, P. PlanarDefect Characteristics and Cross-Sections of $<001\rangle,\langle 111>$, and $<$ 112> InAs Nanowires. I. Appl. Phvs. 2011, 109 (11), 114320.

(17) Conesa-Boj, S.; Kriegner, D.; Han, X.-L.; Plissard, S.; Wallart, X.; Stangl, J.; Fontcuberta i Morral, A.; Caroff, P. Gold-Free Ternary III-V Antimonide Nanowire Arrays on Silicon: Twin-Free down to the First Bilayer. Nano Lett. 2014, 14 (1), 326-332.

(18) Zamani, M. Growth and Characterization of high-quality GaAs nanowires; Master Thesis; Polytecnico di Milano, 2016.

(19) Potts, H.; Morgan, N. P.; Tütüncüoglu, G.; Friedl, M.; Morral, A. F. i. Tuning Growth Direction of Catalyst-Free $\operatorname{InAs}(\mathrm{Sb})$ Nanowires with Indium Droplets. Nanotechnology 2017, 28 (5), 54001.

(20) Li, B.; Yan, X.; Zhang, X.; Ren, X. Self-Catalyzed Growth of InAs Nanowires on InP Substrate. Nanoscale Res. Lett. 2017, 12 (1), 34.

(21) Uccelli, E.; Arbiol, J.; Magen, C.; Krogstrup, P.; Russo-averchi, E.; Heiss, M.; Mugny, G.; Nyg, J.; Morante, J. R.; Fontcuberta, A. Three-Dimensional Multiple-Order Twinning of Self-Catalyzed GaAs Nanowires on Si Substrates. Nano Lett. 2011, 11, 3827-3832.

(22) Fonseka, H. A.; Caroff, P.; Wong-Leung, J.; Ameruddin, A. S.; Tan, H. H.; Jagadish, C. Nanowires Grown on InP (100): Growth Directions, Facets, Crystal Structures, and Relative Yield Control. $\underline{\text { ACS }}$ Nano 2014, 8 (7), 6945-6954.

(23) Matteini, F.; Tutuncuoglu, G.; Potts, H.; Jabeen, F.; Fontcuberta i Morral, A. Wetting of $\mathrm{Ga}$ on $\mathrm{SiOx}$ and Its Impact on GaAs Nanowire Growth. Crust. Growth Des. 2015, 15, 3105.

(24) Matteini, F.; Tutuncuoglu, G.; Mikulik, D.; Vukajlovic-Plestina, J.; Potts, H.; Leran, J. B.; Carter, W. C.; Morral, A. F. Impact of the Ga Droplet Wetting, Morphology, and Pinholes on the Orientation of GaAs Nanowires. Crust. Growth Des. 2016, 16 (10), 5781-5786.

(25) Potts, H.; Friedl, M.; Amaduzzi, F.; Tang, K.; Tütüncüoglu, G.; Matteini, F.; Alarcon Lladó, E.; McIntyre, P. C.; Fontcuberta I Morral, A. From Twinning to Pure Zincblende Catalyst-Free $\operatorname{InAs}(\mathrm{Sb})$ Nanowires. Nano Lett. 2016, 16 (1), 637-643.

(26) Grap, T.; Rieger, T.; Blömers, C.; SchäPers, T.; Grützmacher, D.; Lepsa, M. I. Self-Catalyzed VLS Grown InAs Nanowires with Twinning Superlattices. Nanotechnology 2013, 24 (33), 335601.

(27) Hertenberger, S.; Rudolph, D.; Bolte, S.; Döblinger, M.; Bichler, M.; Spirkoska, D.; Finley, J. J.; Abstreiter, G.; Koblmüller, G. Absence of Vapor-Liquid-Solid Growth during Molecular Beam Epitaxy of Self- 
Induced InAs Nanowires on Si. Appl. Phvs. Lett. 2011, 98 (12), 123114.

(28) Dimakis, E.; Lahnemann, J.; Jahn, U.; Hilse, M.; Geelhaar, L. Self-Assisted Nucleation and Vapor-Solid Growth of InAs Nanowires on Bare Si (111). Crust. Growth Des. 2011, 11, 4001-4008.

(29) Kang, J.-H.; Ronen, Y.; Cohen, Y.; Convertino, D.; Rossi, A.; Coletti, C.; Heun, S.; Sorba, L.; Kacman, P.; Shtrikman, H. MBE Growth of Self-Assisted InAs Nanowires on Graphene. Semicond. Sci. Technol. 2016, 31 (11), 115005.

(30) Madsen, M. H.; Aagesen, M.; Krogstrup, P.; Sørensen, C.; Nygård, J. Influence of the Oxide Layer for Growth of Self-Assisted InAs Nanowires on $\mathrm{Si}(111)$. Nanoscale Res. Lett. 2011, 6 (1), 516.

(31) Oliveira, D. S.; Tizei, L. H. G.; Li, A.; Vasconcelos, T. L.; Senna, C. A.; Archanjo, B. S.; Ugarte, D.; Cotta, M. A. Interaction between Lamellar Twinning and Catalyst Dynamics in Spontaneous Core-shell InGaP Nanowires. Nanoscale 2015, 7 (29), 12722-12727. 